\title{
Improving Wireless Security for Cooperation of Untrusted Secondary Transmitters
}

\author{
Yayan Ma, Baoyun Wang \\ Nanjing University of Posts and Telecommunications \\ 2010010120@njupt.edu.cn,bywang@njupt.edu.cn
}

\begin{abstract}
A modified second-price sealed-bid auction scheme is exploited to study the interaction between the primary transmitter $(P T)$ and multiple untrusted half-duplex secondary transmitters(STS). The STs will eavesdrop on the primary transmission unless they are allowed to transmit with the PT simultaneously. The auction scheme provides an opportunity for intensive cooperation between the PT and untrusted STs which leads to the performance improvement of all the players. In the proposed scheme, a trisection search algorithm is employed to find the optimal bidding power of the STs in the presence of multiple eavesdroppers, and a bisection search algorithm is employed for the optimal transmit power of the winning ST. Numerical results show that the proposed scheme not only improves the achievable secrecy rate of the primary link but also increases the access opportunity of the STs.
\end{abstract}

Keywords: security; auction; eavesdroppers; cognitive radio networks

\section{Introduction}

The notion of secrecy was first proposed by Shannon. In his seminal work [1], Shannon made it possible to obtain the perfect secrecy without any secret key. This notion was applied to the discrete memoryless channel by Wyner[2], with a degraded wiretap channel model. However, when the source-destination channel is worse than the source-eavesdropper channel, the secrecy rate is equal to zero. To overcome this challenge, many researchers focused on potential cooperation among nodes to improve the wireless communication security [3]-[7]. Such a strategy is effective but some selfish nodes are reluctant to consume their resources for the communications which they don't benefit from.

The cooperative strategies for secrecy become perfect in cognitive radio network (CRN)[9], which is a promising technique to alleviate the spectrum shortage by allowing unlicensed users to coexist with licensed users. Many researchers considered a novel cooperation method based on spectrum leasing in which the STs would enhance the security of the primary link by playing the parts of cooperative relays or jammers, in exchange for gaining the spectrum access chances[10]-[12].

The cooperative nodes in the above literatures are trusted. If the nodes are malicious, the cooperation with such nodes becomes complicated [13]-[17], because they are possible to act as eavesdroppers. Game theory is a proper tool to analyze the cooperation of such nodes. There have been several researchers employing game theory to model the cooperation with untrusted nodes [16][17]. A cognitive scenario was studied in [17] where the PT's information must be secured from the untrusted half-duplex STs. In a time slot, the STs can only choose to eavesdrop on the primary traffic or transmit with a fixed power, and they prefer to transmit rather than eavesdrop. The ST who is willing to act as a friendly jammer and improves the secrecy rate best is allowed to access the licensed spectrum by the PT. The author 
modeled the interaction between the PT and untrusted STs as a Stackelberg game in which the PT and STs play the roles of a leader and followers respectively[17]. This scheme takes effect, but it fails to adequately exploit the competitiveness of the multiple STs since they can only transmit with a fixed power.

To tackle this problem, in this paper, a modified second-price sealed-bid auction [18] [19][20], as a type of game theory scheme, is applied to a similar scenario. The main difference between our system model and that of [17] is that the STs in our model can adjust their transmit powers to maximize the achievable secrecy rate for the access opportunities.

The main contributions of our work are summarized as follows:

- The achievable secrecy rate at the primary receiver(PR) is proved to be a quasiconcave function with respect to the winning ST's transmit power if a certain condition is satisfied.

- Two iterative algorithms are proposed: one is based on the trisection search for the optimal bidding power of the STs and the other one is based on the bisection search for the optimal transmit power of the winning ST.

- The proposed auction scheme stimulates the competitiveness of the multiple STs and promotes the cooperation between the PT and untrusted STs, so it brings the simultaneous performance improvement for all the players.

The remainder of the paper is organized as follows. In Section 2, the system model is presented. In Section 3, we study the cooperation between the PT and multiple untrusted STs using the modified second-price sealed-bid auction scheme. Simulation results are provided in Section 4, followed by the conclusion in Section 5.

\section{System Model}

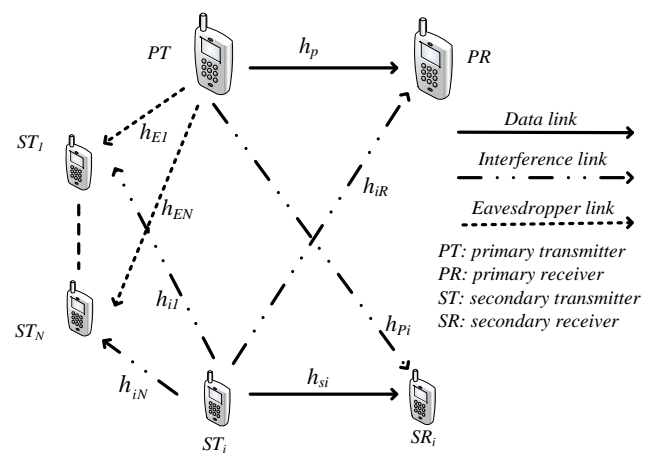

Figure 1. System Model

We consider a CRN with one primary pair and multiple secondary pairs $(i=1, \ldots, N)$ as illustrated in Fig.1. The STs are untrusted and their communication mode is half-duplex, in other words, they will eavesdrop on the primary link when they are not allowed to access the licensed spectrum. It's assumed that the STs prefer to transmit rather than eavesdrop and they do not collude. It's easy to see that the secrecy rate equals zero when the primary link is worse than the eavesdropper link. In our model the receivers consider the information from unintended transmitters as interference. If an appropriate ST is selected by the PT to transmit simultaneously, it causes less interference to the PR than to the unselected STs, and the security of the primary link is enhanced.

Denote the instantaneous channel gain between the PT and the PR as $h_{p}$, between the ST $i$ and the ST $j$ as $h_{i j}$, between the PT and the ST $i$ as $h_{E i}$. Similarly, we 
define $h_{s i}, h_{P i}, h_{i R}$, as illustrated in Fig.1. The transmit powers for the ST $i$ and the PT are $P_{s i}$ and $P_{p}$. The maximum transmit power for all the nodes is $P^{\max }$. The noise power is assumed to be the same for all the links, denoted by $\sigma^{2}$.

If the PT selects the ST $i$ to cooperate, other unselected STs act as eavesdroppers, the achievable secrecy rate of the primary link in the presence of the ST $j$ is:

$$
R_{s}^{i}\left(P_{s i}, j\right)=\left[\log \left(1+\frac{P_{p} h_{p}}{\sigma^{2}+P_{s i} h_{i R}}\right)-\log \left(1+\frac{P_{p} h_{E j}}{\sigma^{2}+P_{s i} h_{i j}}\right)\right]^{+} \square
$$

where $[x]^{+}=\max (x, 0)$.

The achievable secrecy rate of the primary link in the presence of all the eavesdroppers (i.e. all unselected STs) is:

$$
R_{s}^{i}\left(P_{s i}\right)=\min _{j \neq i}\left[\log \left(1+\frac{P_{p} h_{p}}{\sigma^{2}+P_{s i} h_{i R}}\right)-\log \left(1+\frac{P_{p} h_{E j}}{\sigma^{2}+P_{s i} h_{i j}}\right)\right]^{+}
$$

The achievable rate of the secondary pair $i$ is expressed as:

$$
R_{s i}\left(P_{s i}\right)=\log \left(1+\frac{P_{s i} h_{s i}}{\sigma^{2}+P_{p} h_{P i}}\right)
$$

\section{Auction Scheme}

\subsection{Game Theory Model}

In this paper, all the nodes are assumed to be selfish and rational. Game theory is a proper mathematical tool to analyze the interaction of such nodes [18]. As a kind of game theory, auction is a mature scheme to analyze how to assign resource efficiently. When a sealed-bid auction is exploited, the privacy of bidders is well guarded and the bidders submit bids simultaneously. Among various sealed-bid auction schemes, a second-price sealed-bid auction is famous for its truthful property, namely, each bidder will bid with the maximum amount it would like to pay for the object. In a second-price sealed-bid auction scheme, the bidder who submits the highest bid is rewarded with the object, only charged the second highest bid. If the bidder bids with a value less than its own valuation for the object, its winning chance is reduced. If it would pay the auctioneer larger than its own valuation, the winning chance increases but its utility is negative. The dominant strategy equilibrium (DSE), in which each player finds its perfect strategy regardless what other players may play, corresponds to such truthful strategy [18]. This means every bidder has no demand for the information on other bidders in a second-price sealed-bid auction.

In short, we model the cooperation between the PT and untrusted STs as a second-price sealed-bid auction in which the PT and untrusted STs are the auctioneer and bidders respectively, and the ST who provides the PT with the largest secrecy rate wins the access chance of the licensed spectrum.

The strategy of each bidder is its transmit power choice. If the bidder $i$ is selected by the PT, its utility is given as follows:

$$
U_{s i}\left(P_{s i}\right)=\left[\log \left(1+\frac{P_{s i} h_{s i}}{\sigma^{2}+P_{p} h_{P i}}\right)-c_{s i} P_{s i}\right]^{+}
$$

where $c_{s i}$ is defined as the cost per unit transmission power. Otherwise the bidder's utility is zero. 
The bidder $i$ 's bid is the achievable secrecy rate of the primary link given by (2). It means that the parameters: $h_{p}$ and $h_{E i}(i=1, \ldots, N)$ must be provided to the bidder $i$. The reserve price $R_{s}^{0}$ set by the auctioneer is:

$$
R_{s}^{0}=\min _{i=1, \ldots, N}\left[\log \left(1+\frac{P_{p} h_{p}}{\sigma^{2}}\right)-\log \left(1+\frac{P_{p} h_{E i}}{\sigma^{2}}\right)\right]^{+}
$$

Only the bid which is larger than $R_{s}^{0}$ can be accepted by the auctioneer.

Denoting the strategy of the bidder $i$ in the DSE as $P_{s i}^{\text {bid }}$, the index of the winning bidder is

$$
w=\arg \max _{i=1 . . . N} R_{s}^{i}\left(P_{s i}\right)
$$

if $R_{s}^{w}\left(P_{s w}\right)>R_{s}^{0}$, otherwise no bidder is selected by the auctioneer. In the case that there are multiple equal highest bids, the auctioneer selects the cooperative bidder randomly.

The winning bidder will be allowed to access the licensed spectrum only required to provide the auctioneer with the second largest bid which is defined as follows:

$$
R_{s}^{2}=\max _{i \neq w}\left(\max \left(R_{s}\left(P_{s i}, i\right), R_{s}^{0}\right)\right.
$$

\subsection{Equilibrium}

It's clear that $U_{s i}\left(P_{s i}\right)\left(P_{s i} \geq 0\right)$ is a concave function in $P_{s i}$ and will be larger than zero over the range of $0 \leq P_{s i} \leq P_{s i}^{0}\left(P_{s i}^{0}\right.$ is the nonzero solution of $\left.U_{s i}\left(P_{s i}\right)=0\right)$, if the condition

$$
d_{s i}=\left.\frac{\partial U_{s i}}{\partial P_{s i}}\right|_{P_{s i}=0}>0
$$

is satisfied. Only this condition is met, the bidder $i$ is willing to participate in the bidding.

Define

$$
\begin{aligned}
& k=\arg \max _{i=1 . \ldots N}\left(h_{E i}\right) \\
& b=\arg \max _{i \neq k}\left(h_{E i}\right) \\
& K= \begin{cases}k & i \neq k \\
b & i=k\end{cases}
\end{aligned}
$$

then the ST $K$ 's eavesdropper channel gain is the largest among that of unselected STs when the ST $i$ is selected by the PT to cooperate.

As mentioned above, the bidder $i$ (i.e. the ST $i$ ) will improve the PT's secrecy rate as far as possible to maximize its winning chance, so its dominant strategy $P_{s i}^{\text {bid }}$ is the solution to the following optimization problem :

$$
\begin{aligned}
P_{s i}^{b i d}= & \arg \max _{P_{s i}} R_{s}^{i}\left(P_{s i}\right) \\
& =\arg \max _{P_{s i}} \min _{j \neq i} R_{s}^{i}\left(P_{s i}, j\right) \\
\text { s.t. } & 0 \leq P_{s i} \leq P_{s i}^{\lim } \\
& R_{s}^{i}\left(P_{s i}\right)>R_{s}^{0}
\end{aligned}
$$

where $R_{s}^{i}\left(P_{s i}, j\right), R_{s}^{i}\left(P_{s i}\right)$ and $R_{s}^{0}$ are defined as (1), (2) and (5) respectively, $P_{s i}^{\lim }$ is given as follows

$$
P_{s i}^{\lim }=\min \left(P_{s i}^{0}, P^{\max }\right)
$$

The bid $R_{s i}{ }_{s i}$ of the $\mathrm{ST} i$ is also given as follows: 


$$
R_{s i}^{b i d}=R_{s}^{i}\left(P_{s i}^{b i d}\right)
$$

In order to solve this optimization problem, we give the property of the function $R_{s}^{i}\left(P_{s i}\right)$ in Theorem 1.

Theorem 1. The function of the secrecy rate $R_{s}^{i}\left(P_{s i}\right)$ is quasi-concave in $P_{s i}$ in the range $P_{s i} \geq 0$ and can achieve a value larger than $R_{s}{ }^{0}$, iff one of the following conditions is satisfied:

$$
\begin{gathered}
\mathrm{A}_{i}^{K}>0, \mathrm{C}_{i}^{K}<0, P_{s i}^{\lim }>\max \left(-B_{i}^{K} / 2 \mathrm{~A}_{i}^{K}, 0\right) \\
i=k, \mathrm{C}_{i}^{K}>0, B_{i}^{K}>0
\end{gathered}
$$

where $R_{s}^{0}, k$ and $K, P_{s i}^{\lim }$ are given by (5), (9) and (11),(13) respectively,

$$
\begin{aligned}
& A_{i}^{K}=h_{i K} h_{i R}\left(h_{i K} h_{p}-h_{E K} h_{i R}\right), B_{i}^{K}=2 h_{i K} h_{i R} \sigma^{2}\left(h_{p}-h_{E K}\right), \\
& C_{i}^{K}=\sigma^{4}\left(h_{i R} h_{p}-h_{E K} h_{i K}\right)+\sigma^{2} h_{p} h_{E K} P_{p}\left(h_{i R}-h_{i K}\right) .
\end{aligned}
$$

Proof: Please see Appendix A.

Due to the properties of the functions $R_{s}^{i}\left(P_{s i}\right)$ and $U_{s i}\left(P_{s i}\right)$, the dominant strategy $P_{s i}^{b i d}$ in the DSE, as the solution of the optimization problem (12), can be obtained by the trisection search. The detailed steps are presented in Algorithm 1.

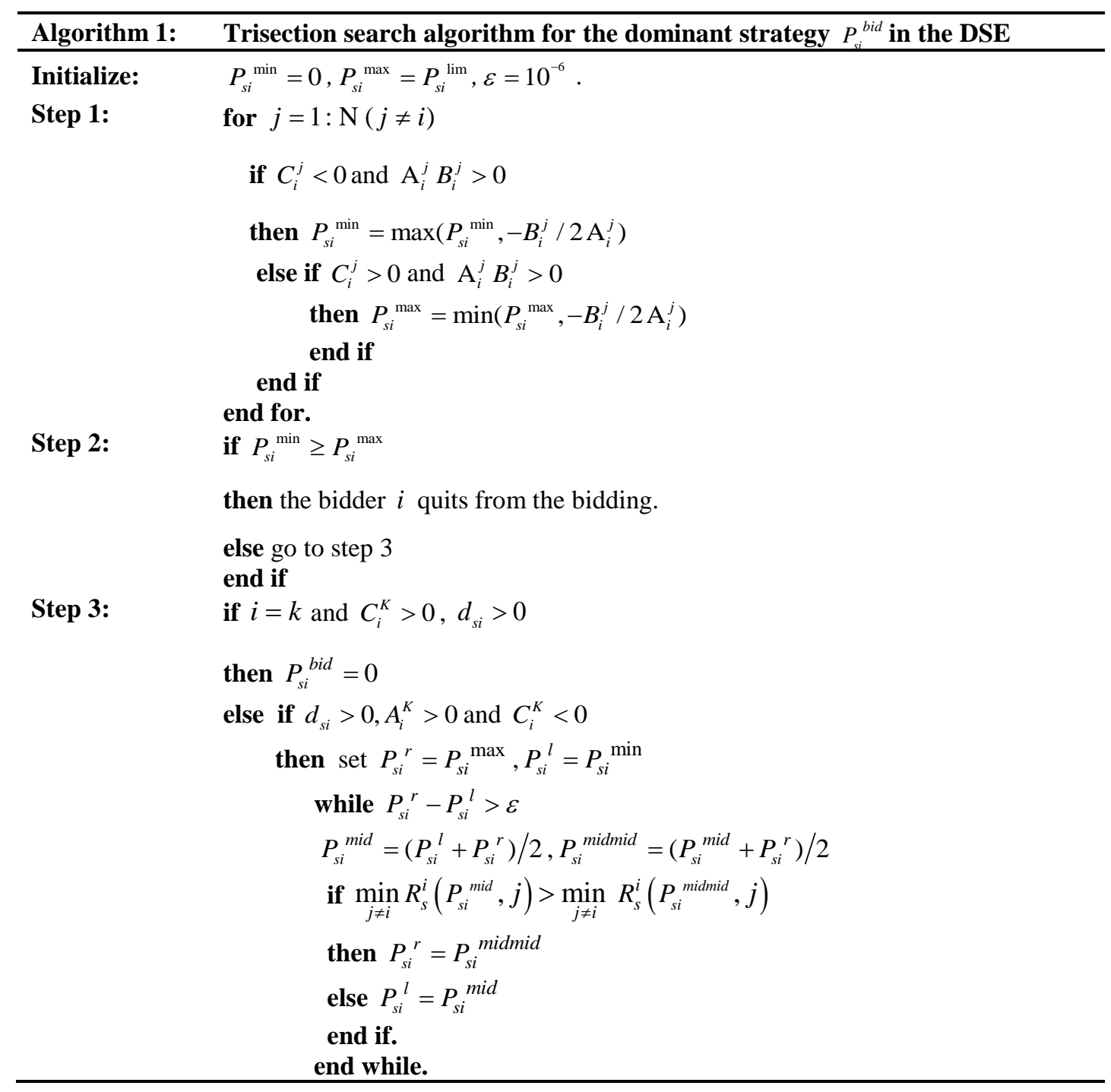




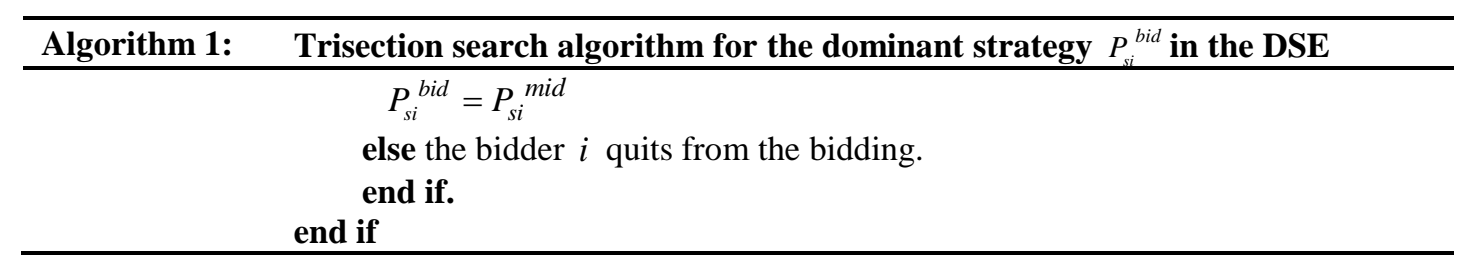

\subsection{The Power of the Winning ST}

The index of the winning bidder $w$ is defined as (6). If the traditional auction theory is employed, the relationship between the utility of the auctioneer and that of the winning bidder is opposite, namely one increases while the other decreases. But, in our second-price sealed-bid auction model, the utilities of the auctioneer and winning bidder may increase or decrease simultaneously in some specific ranges of $P_{s w}$ due to their concavity. Therefore we slightly modify the second-price sealed-bid auction scheme [11]: the winning bidder must provide at least the second highest bid. In this setting, the winning ST $w$ may maximize its own utility by adjusting the transmit power $P_{s w}$ under the constraint $R_{s}^{w}\left(P_{s w}\right) \geq R_{s}{ }^{2}$, which can bring the simultaneous performance improvement to the PT and winning ST.

The outcome of the modified second-price sealed-bid auction is summed up in Theorem2.

Theorem 2 The optimum transmit power $P_{s w}{ }^{*}$ of the winning secondary transmitter $w$ is:

$$
P_{s w}{ }^{*}= \begin{cases}P^{S H} & P_{s w}{ }^{o p t}>P^{S H}, R_{s}^{2}>0 \\ P^{S L} & P_{s w}^{o p t}<P^{S L}, R_{s}^{2}>0 \\ P_{s w}{ }^{o p t} & P^{S L} \leq P_{s w}^{o p t} \leq P^{S H}, R_{s}^{2}>0 \\ P_{s w}{ }^{o p t} & R_{s}{ }^{2}=0\end{cases}
$$

where $P^{S H}$ and $P^{S L}\left(P^{S H}>P^{S L}\right)$ are the transmit powers with which the winning secondary transmitter can provide the primary transmitter with the second highest bid $R_{s}^{2}$, and their values are given by Algorithm 2; $P_{s w}^{o p t}$ is the transmit power which maximizes the winning secondary transmitter's utility and is expressed as Error! Reference source not found.:

$$
P_{s w}^{o p t}=\left[\frac{1}{c_{s w} \ln 2}-\frac{P_{p} h_{P_{w}}+\sigma^{2}}{h_{s w}}\right]_{0}^{p^{p m a x}}
$$

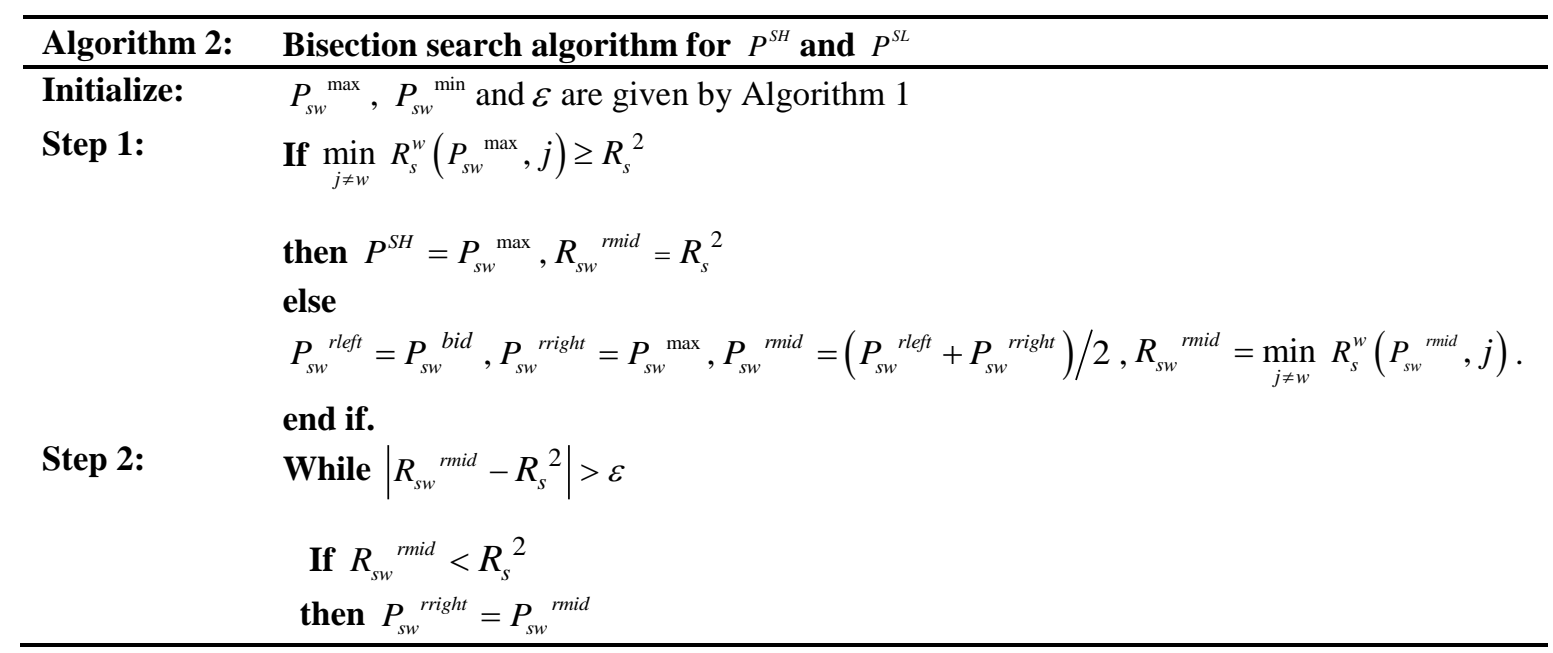




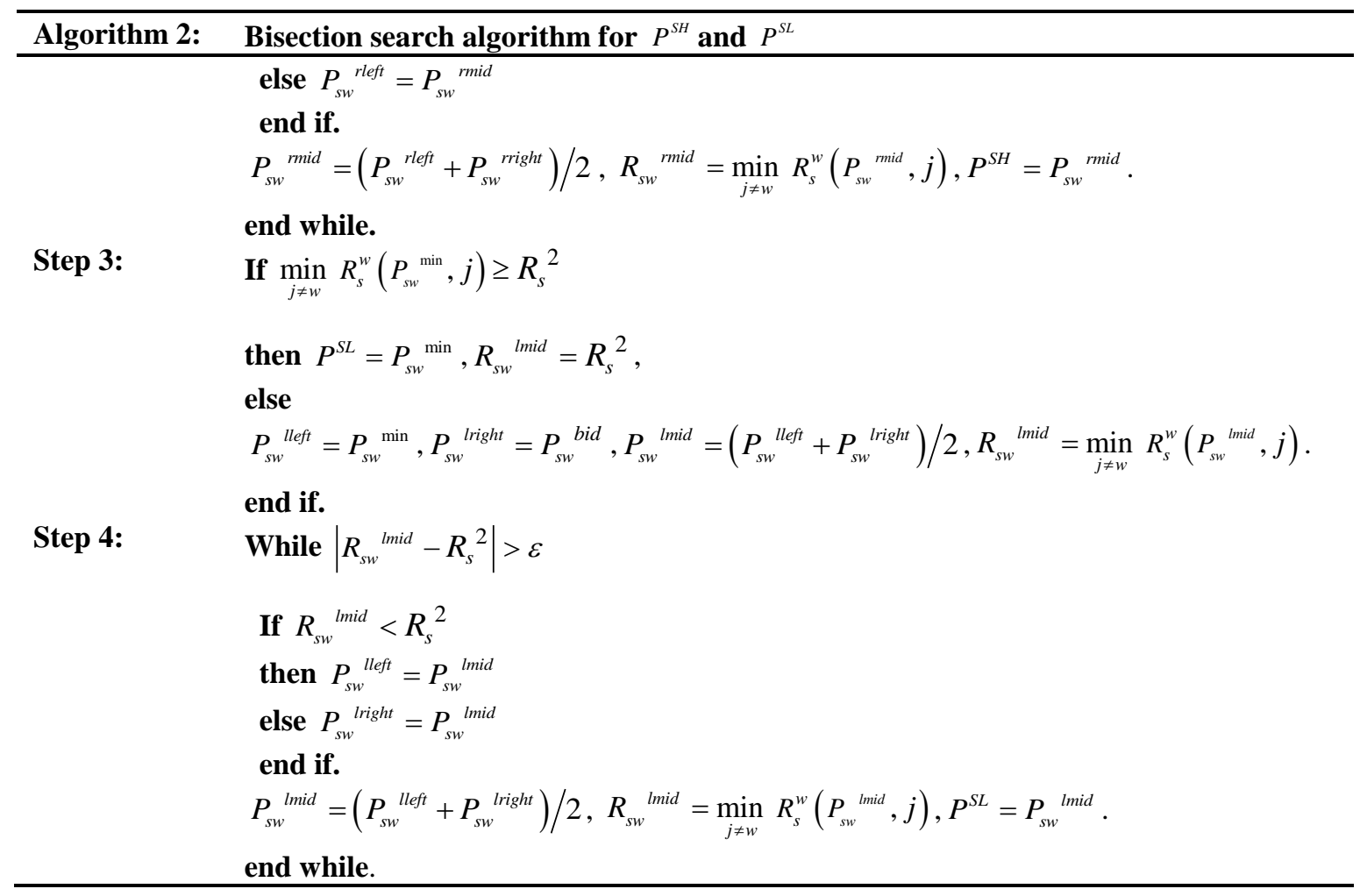

Proof: According to Theorem 1 and Algorithm 1, the function of the secrecy rate $R_{s}^{w}\left(P_{s w}\right)$ is increasing in the region $P^{S L} \leq P_{s w} \leq P_{s w}^{b i d}$ and decreasing in the region $P_{s w}{ }^{b i d} \leq P_{s w} \leq P^{S H}$. If the transmit power $P_{s w}{ }^{s}$ selected by the winning ST lies in the range of $P^{S L} \leq P_{s w}{ }^{s} \leq P^{S H}$, then $R_{s}{ }^{2} \leq R_{s}^{w}\left(P_{s w}{ }^{s}\right)<R_{s w}{ }^{b i d}$. As mentioned above, the winning ST $w$ will selected an optimum transmit power to maximize its own utility on the basis of ensuring that the secrecy rate $R_{s}^{w}\left(P_{s w}\right)$ is greater than or equal to $R_{s}^{2}$, and formula Error! Reference source not found. just gives such a solution. The proof is completed.

It can be observed from Theorem 2 that if the condition $P^{S L} \leq P_{s w}{ }^{o p t} \leq P^{S H}$ is satisfied, the alteration of the auction scheme not only maximizes the winning ST's utility but also increases the secrecy rate from $R_{s}{ }^{2}$ to $R_{s}^{w}\left(P_{s w}{ }^{o p t}\right)$. Otherwise, the winning $\mathrm{ST}$ will provide the PT with the secrecy rate $R_{s}^{2}$, and its own utility is $U_{s w}\left(P^{S L}\right)$ (or $U_{s w}\left(P^{S H}\right)$ ) which is less than $U_{s w}\left(P_{s w}{ }^{o p t}\right)$.

\section{Numerical Results}

In this section, numerical results are provided to demonstrate the proposed second-price sealed-bid auction scheme for our system model. A simple model is considered in which the STs are all scattered at about the same normalizing-distance from the PT and PR. We consider a flat Rayleigh fading environment, in which the averaged power channel gains are set by $E\left[h_{p}\right]=E\left[h_{s i}\right]=E\left[h_{E i}\right]=1$, $E\left[h_{i j}\right]=E\left[h_{i R}\right]=E\left[h_{P i}\right]=0.1$, the noise power $\sigma^{2}, P_{p}$ and $P^{\max }$ are $1 \mathrm{mw}, 10 \mathrm{mw}$ and $10 \mathrm{mw}$, respectively. The value of the cost per unit transmission power $c_{s i}$ is shown in the figures. Every result is obtained by $10^{5}$ trials. 
The average achievable secrecy rate of the primary link and the average utility of the winning ST are illustrated in Figure 2 and Figure 3 respectively, as functions of the number of the STs $N$, using the Stackelberg scheme proposed in [17] and our proposed modified second-price sealed-bid auction scheme.

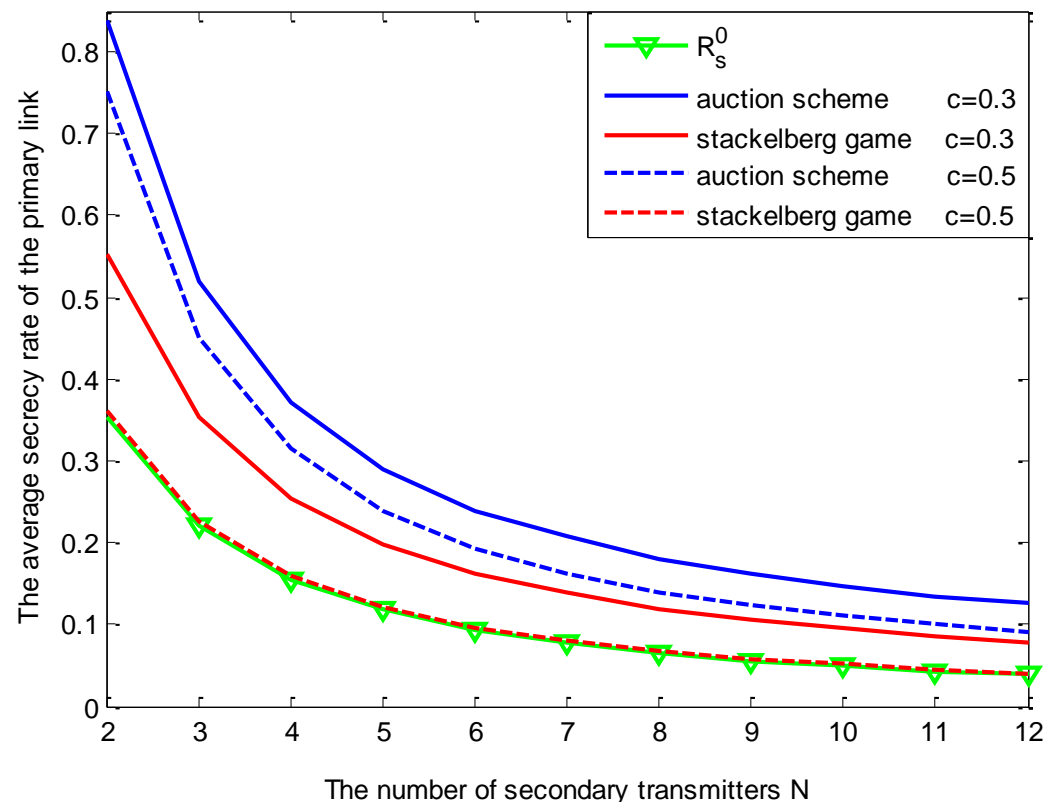

Figure 2. The Average Secrecy Rate of the Primary Link Versus the Number of Secondary Transmitters

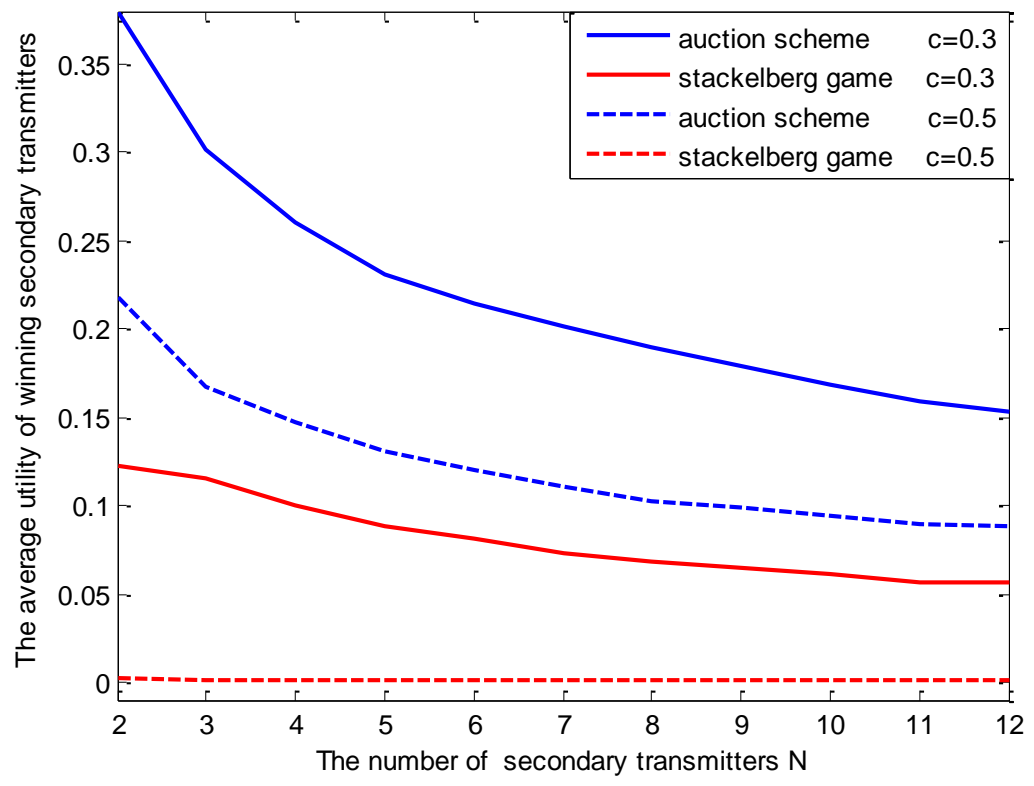

Figure 3. The Average Utility of the Winning Secondary Transmitter Versus the Number of Secondary Transmitters

From Figure 2 and Figure 3, it is observed that both the average secrecy rate and the average utility of the winning ST benefit from these two schemes, compared with the case that the PT and STs do not cooperate. It should be noticed that the 
utility of each unselected ST is zero. Due to competitiveness, our proposed scheme can improve the average secrecy rate better than the Stackelberg scheme as shown in Figure 2. The similar relation of both schemes holds for the utility of the winning ST as our proposed auction scheme increases its access opportunity better. The advantage of the two schemes dwindles with the increase of the number $N$ of STs because the number of the potential eavesdroppers increases.

If the Stackelberg scheme is employed, the winning ST has to passively choose to eavesdrop on the primary link or access the licensed spectrum with a fixed power $P^{\max }$. For an enough large $c_{s i}$ (the cost per unit transmission power), the utility of the winning ST is negative, so it isn't willing to transmit. In such a case the performance of all the nodes could be hardly improved by the Stackelberg scheme as shown in Figure 2 and Figure 3 when $c_{s i}=0.5$.

Figure 4 demonstrates the failure probability of cooperation between the PT and all the STs versus the number $N$ for the above two schemes. If the Stackelberg scheme is employed and $c_{s i}=0.5$, the failure probability of cooperation approaches one, which means almost all the STs choose to eavesdrop in such case. It is clear that our auction scheme provides better cooperation between the PT and untrusted STs than the Stackelberg scheme.

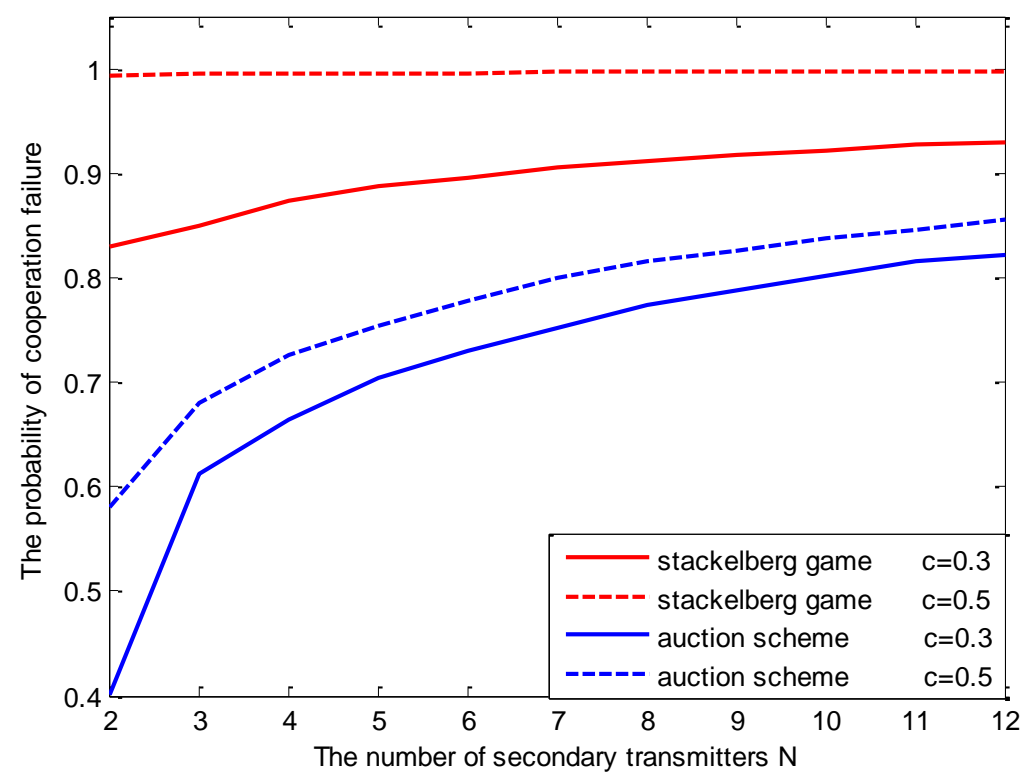

\section{Figure 3. The Failure Probability of Cooperation between the PT and STs Versus the Number of Secondary Transmitters}

\section{Conclusion}

In this paper, we employed a modified second-price sealed-bid auction scheme to model the interaction between the PT and multiple untrusted half-duplex STs. If the STs are not permitted to access the licensed spectrum by the PT, they will act as the eavesdroppers on the primary link. The proposed auction scheme stimulates the competitiveness of the multiple STs and promotes the cooperation between the PT and untrusted STs, which improves the performance of all the participants. Two iterative algorithms were proposed: one is based on the trisection search for the optimal bidding power of the STs, and the other one is based on the bisection search for the optimal transmit power of the winning ST. Numerical results corroborate that the 
proposed scheme not only enhances the security of the primary link but also increases the STs' access chance.

\section{Acknowledgment}

This work was supported by National Natural Science Foundation of China (Grant No 61271232, 61372126), the University Postgraduate Research and Innovation Project in Jiangsu Province (CXZZ12-0472).

\section{Appendix A}

Firstly, we prove that the function $R_{s}^{i}\left(P_{s i}, j\right)$ is quasi-concave for $P_{s i} \geq 0$ iff one of the conditions Error! Reference source not found. and Error! Reference source not found. is satisfied.

Define $F_{s}^{i}\left(P_{s i}, j\right)=\log \left(1+\frac{P_{p} h_{p}}{\sigma^{2}+P_{s i} h_{i R}}\right)-\log \left(1+\frac{P_{p} h_{E j}}{\sigma^{2}+P_{s i} h_{i j}}\right)$, then $R_{s}^{i}\left(P_{s i}, j\right)=\left[F_{s}^{i}\left(P_{s i}, j\right)\right]^{+}$.

Take the derivative of $F_{s}^{i}\left(P_{s i}, j\right)$ with respect to $P_{s i}$ and set it to zero, we'll obtain:

$$
\mathrm{A}_{i}^{j} P_{s i}^{2}+B_{i}^{j} P_{s i}+C_{i}^{j}=0
$$

The equation Error! Reference source not found. has two solutions as follows

$P_{s i}(1)=\frac{-B_{i}^{j}+\sqrt{B_{i}^{j 2}-4 \mathrm{~A}_{i}^{j} C_{i}^{j}}}{2 \mathrm{~A}_{i}^{j}}$

$P_{s i}(2)=\frac{-B_{i}^{j}-\sqrt{B_{i}^{j 2}-4 \mathrm{~A}_{i}^{j} C_{i}^{j}}}{2 \mathrm{~A}_{i}^{j}}$

If $\mathrm{A}_{i}^{j}>0, C_{i}^{j}<0$, we have $P_{s i}(1)>0, P_{s i}(2)<0$ and $\partial F_{s}^{i}\left(P_{s i}, j\right) /\left.\partial P_{s i}\right|_{P_{s i}=0}>0$.Further, when $P_{s i} \rightarrow \infty, F_{s}^{i}\left(P_{s i}, j\right) \rightarrow 0$. So $F_{s}^{i}\left(P_{s i}, j\right)$ is an continuous increasing function of the transmit power $P_{s_{i}}$ in the range of $\left[0, P_{s i}(1)\right]$ and a continuous decreasing one in the range of $\left[P_{s i}(1),+\infty\right]$. According to [21], we can claim that the function $F_{s}^{i}\left(P_{s i}, j\right)$ is quasiconcave in $P_{s i}$ for $P_{s i} \geq 0$. For $\partial F_{s}^{i}\left(P_{s i}, j\right) /\left.\partial P_{s i}\right|_{P_{s i}=0}>0$, the value of $F_{s}^{i}\left(P_{s i}, j\right)$ larger than $F_{s}^{i}(0, j)$ will be achieved for $P_{s i}>0$. Let $F_{s}^{i}\left(P_{s i}, j\right)>0$, we can obtain $P_{s i}>-B_{i}^{j} / 2 \mathrm{~A}_{i}^{j}$. In short, $R_{s}^{i}\left(P_{s i}, j\right)$ is a quasi-concave function for $P_{s i} \geq 0$ and it will achieve a value larger than $R_{s}^{i}(0, j)$ in the range $P_{s i}>\max \left(-B_{i}^{j} / 2 \mathrm{~A}_{i}^{j}, 0\right)$.

Similarly, if $\mathrm{C}_{i}^{K}>0, B_{i}^{K}>0, R_{s}^{i}\left(P_{s i}, j\right)$ is proved to be a continuous decreasing function of $P_{s_{i}}$ for $P_{s i}>0$. In such a case, if $i=k$,i.e., the ST $i$ 's eavesdropper channel gain is larger than that of other STs, the function $R_{s}^{i}\left(P_{s i}, j\right)$ will have a value larger than $R_{s}{ }^{0}$ for $P_{s i} \geq 0$.

To sum up, we prove that the function $R_{s}^{i}\left(P_{s i}, j\right)$ is quasi-concave in $P_{s i}$ for $P_{s i} \geq 0$ and will achieve a value larger than $R_{s}{ }^{0}$, iff one of the conditions Error! Reference source not found. and Error! Reference source not found. is satisfied.

Next, due to the quasi-concavity preservation, $R_{s}^{i}\left(P_{s i}\right)\left(\right.$ i.e. $\left.\min _{j \neq i} R_{s}^{i}\left(P_{s i}, j\right)\right)$ is also a quasi-concave function for $P_{s i} \geq 0$ and can achieve a value larger than $R_{s}{ }^{0}$, iff one of the conditions Error! Reference source not found. and Error! Reference source not found. is satisfied.

The proof is completed. 


\section{References}

[1] Shannon C E., "Communication theory of secrecy systems," Bell System Technical Journal (1949), Vol.28, No.4, pp. $656-715$.

[2] A. D.Wyner, “The wire-tap channel,” Bell System Technical Journal (1975), Vol. 54, No.8, pp. 1355 1387.

[3] A. Mukherjee, A. Swindlehurst, "Jamming games in the MIMO wiretap channel with an active eavesdropper," IEEE Trans.on Signal Process ( 2013), Vol. 61, No.1, pp. 82 - 91.

[4] J. Chen, R. Zhang, L. Song, and Z. H. B. Jiao, "Joint relay and jammer selection for secure two-way relay networks," IEEE Trans. on Information Forensics Security( 2012), Vol.7, No.1, pp. 310 - 320.

[5] L. Lai and H. El Gamal, "The relay-eavesdropper channel: Cooperation for secrecy," IEEE Trans. on Information Theory (2008), Vol.54, No.9, pp. 4005 - 4019.

[6] E. Tekin and A. Yener, "The general Gaussian multiple-access and twoway wiretap channels: Achievable rates and cooperative jamming," IEEETrans. on Information Theory(2008) ,Vol.54, No.6, pp. 2735 - 2751.

[7] X. Tang, R. Liu, P. Spasojevic, and H. V. Poor, "Interference assisted secret communication," IEEE Trans. on Information Theory (2011), Vol.57, No.5, pp. 3153 - 3167.

[8] L. Dong, Z. Han, A. Petropulu, and H. V. Poor, "Improving wireless physical layer security via cooperating relays," IEEE Trans. on Signal Process ( 2010),Vol.58, No.3, pp. 1875 - 1888.

[9] A. Goldsmith, S. A. Jafar, I. Maric, and S. Srinivasa, "Breaking spectrum gridlock with cognitive radios: an information theoretic perspective,"Proc. IEEE (2009), Vol.97, No.5, pp. 894 - 914..

[10] K. Lee, O. Simeone, C. Chae, and J. Kang, "Spectrum leasing via cooperation for enhanced physicallayer secrecy," in Proc. IEEE International Conference on Communications (ICC),(2011) June 5-9; Kyoto, Japan

[11] Stanojev, Igor, and Aylin Yener., "Improving secrecy rate via spectrum leasing for friendly jamming," IEEE Trans. on Wireless Communications (2013),Vol.12, No.1, pp. 134 - 145.

[12] Zhang, N., Lu, N., Cheng, N., Mark, J. W., \& Shen, X. S,. "Cooperative spectrum access towards secure information transfer for crns," IEEE Journal on. Selected Areas in Communications (2013) ,Vol.31, No.11, pp. 2453 - 2464.

[13] X. He and A. Yener, "Cooperation with an untrusted relay: A secrecy perspective," IEEE Trans. on Information Theory (2010), Vol.56, No.8, pp. 3807 - $3827 .$.

[14] M. Yuksel, X. Liu, and E. Erkip, “A secure communication game with a relay helping the eavesdropper," IEEE Trans. on Information Forens. Security (2011),Vol.6, No.3, pp. 818 - 830..

[15] Hyoungsuk Jeon., Steven W. McLaughlin., Il-Min Kim and Jeongseok Ha, "Secure Communications with Untrusted Secondary Nodes in Cognitive Radio Networks,", IEEE Transactions on. Wireless Communications (2014), Vol.13, No.4, pp. 1790 - 1805.

[16] Frédéric Gabry., Nan Li, Nicolas Schrammar., Maksym Girnyk, Lars K. Rasmussen, Mikael Skoglund," On the Optimization of the Secondary Transmitter's Strategy in Cognitive Radio Channels with Secrecy," IEEE Journal on. Selected Areas in Communications (2014) ,Vol.32, No.3, pp. 451 - 463.

[17] Karim Khalil and Eylem Ekici, "Secrecy in cognitive radio networks: Turning foes to allies," in Proc. Modeling \& Optimization in Mobile, Ad Hoc \& Wireless Networks (WiOpt), (2013) May 13-17; Tsukuba Science City, Japan.

[18] P. Klemperer, "Auction Theory: A Guide to the Literature," Journal of Economic Surveys, (1999) ,Vol.13, No.3, pp. 227-286.

[19] Tianyu Wang, Lingyang Song, Zhu Han, Xiang Cheng, and Bingli Jiao, "Power Allocation using Vickrey Auction and Sequential First-Price Auction Games for Physical Layer Security in Cognitive Relay networks", in Proc. International Conference on Communications (ICC),(2012) June 10-15; Ottawa, Canada.

[20] M. J. Osborne and A. Rubenstein, in A Course in Game Theory, MIT Press, Massachusetts (1994), Vol. 4, pp18-30.

[21] Stephen Boyd and Lieven Vandenberghe, in Convex Optimization,Cambridge University Press, Cambridge (2004), Vol. 6 pp95-103. 


\begin{abstract}
Authors
Yayan Ma is pursuing Doctorate degree at Nanjing University of Posts and Telecommunications. Her current research interests include signal processing, game theory, and its applications to physical security.
\end{abstract}
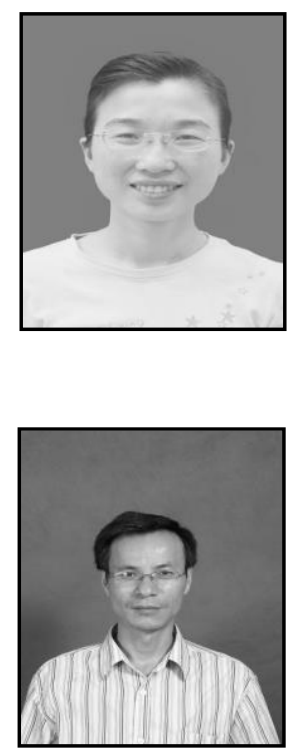

Baoyun Wang is a professor at Nanjing University of Posts and Telecommunications. Her current research interests include information security, signal processing, game theory, and its applications to physical security. 